\title{
BMJ Open Better evidence for earlier assessment and surgical intervention for refractory epilepsy (The BEST study): a mixed methods study protocol
}

\author{
Frances Rapport, ${ }^{1}$ Patti Shih, ${ }^{1}$ Rebecca Mitchell, ${ }^{1}$ Armin Nikpour, ${ }^{2,3}$ \\ Andrew Bleasel, ${ }^{3,4}$ Geoffrey Herkes, ${ }^{3,5}$ Sanjyot Vagholkar, ${ }^{6}$ Virginia Mumford ${ }^{1}$
}

To cite: Rapport F, Shih $P$, Mitchell $\mathrm{R}$, et al. Better evidence for earlier assessment and surgical intervention for refractory epilepsy (The BEST study): a mixed methods study protocol. BMJ Open 2017;7:e017148. doi:10.1136/ bmjopen-2017-017148

- Prepublication history for this paper is available online. To view these files please visit the journal online (http://dx.doi. org/10.1136/bmjopen-2017017148).

Received 5 April 2017 Revised 23 June 2017 Accepted 6 July 2017

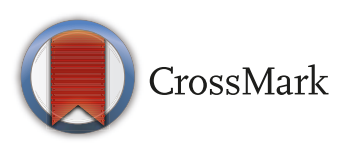

${ }^{1}$ Faculty of Medicine and Health Sciences, Australian Institute of Health Innovation, Macquarie University, Sydney, Australia ${ }^{2}$ Royal Prince Alfred Hospital, Sydney, Australia

${ }^{3}$ University of Sydney, Sydney, Australia

${ }^{4}$ Westmead Hospital, Westmead, Australia

${ }^{5}$ Royal North Shore Hospital,

Sydney, Australia

${ }^{6}$ Faculty of Medicine and Health Sciences, Primary Care \&

Wellbeing, Macquarie University, Sydney, Australia

Correspondence to

Professor Frances Rapport;

frances.rapport@mq.edu.au

\section{ABSTRACT}

Introduction One-third of patients with refractory epilepsy may be candidates for resective surgery, which can lead to positive clinical outcomes if efficiently managed. In Australia, there is currently between a 6-month and 2-year delay for patients who are candidates for respective epilepsy surgery from the point of referral for surgical assessment to the eventual surgical intervention. This is a major challenge for implementation of effective treatment for individuals who could potentially benefit from surgery. This study examines implications of delays following the point of eligibility for surgery, in the assessment and treatment of patients, and the factors causing treatment delays.

Methods and analysis Mixed methods design: Observations of qualitative consultations, patient and healthcare professional interviews, and health-related quality of life assessments for a group of 10 patients and six healthcare professionals (group 1); quantitative retrospective medical records' reviews examining longitudinal outcomes for 50 patients assessed for, or undergoing, resective surgery between 2014 and 2016 (group 2); retrospective epidemiological study of all individuals hospitalised with a diagnosis of epilepsy in New South Wales (NSW) in the last 5 years (2012-2016; approximately 11000 hospitalisations per year, total 55 $000)$, examining health services' use and treatment for individuals with epilepsy, including refractory surgery outcomes (group 3).

Ethics and dissemination Ethical approval has been granted by the North Sydney Local Health District Human Research Ethics Committee (HREC/17/HAWKE/22) and the NSW Population \& Health Services Research Ethics Committee (HREC/16/CIPHS/1). Results will be disseminated through publications, reports and conference presentations to patients and families, health professionals and researchers.

\section{INTRODUCTION}

\section{Underutilisation of resective surgery}

Refractory epilepsy, where seizures cannot be controlled with seizure medication, is responsible for most of the burden of disease (BoD) in epilepsy, ${ }^{1}$ leading to high rates of

\section{Strengths and limitations of this study}

- First Australian study examining implications in delay for early identification, assessment and treatment of patients with refractory epilepsy eligible for resective surgery.

- More detailed understanding of challenges facing practitioners working in complex epilepsy.

- Unique combination of data capture events, including a comprehensive epidemiological examination alongside a year-long qualitative assessment of patient journeys through the healthcare system across key New South Wales clinics.

- The level of detail disclosed lends itself to transferability of findings to other Australian jurisdictions, and international healthcare services.

- Small-scale qualitative investigation, with only two neurology clinics involved, yet depth of qualitative data capture assures a rich dataset that complements the epidemiological investigation and ensures a clear design structure for a larger-scale, pan-Australia intervention study, promising more clinics and greater socioeconomic patient diversity.

comorbidity, decreased life expectancy, ${ }^{2}{ }^{3}$ stigmatisation, reduced health-related quality of life (HRQoL) and extensive psychosocial problems. ${ }^{4}$ For adults living with epilepsy, there is increased risk of depression and anxiety, difficulty in developing and maintaining relationships and employment issues, as well as experiences of multiple phobias. ${ }^{5}$

Within the refractory epilepsy group, one-third of patients who are unresponsive to drugs and remain with an uncontrollable disease may be candidates for resective surgery. ${ }^{1}$ Resective epilepsy surgery can be effective for many people, and while curative epilepsy surgery does require a single onset zone that can be resected safely, there are palliative surgical procedures where the focus cannot be found, or there are multiple foci of onset). A third of patients with refractory 
epilepsy may be surgery candidates ${ }^{67}$ and while some continue with seizures, or seizures return, ${ }^{12}$ the majority, particularly among adult patients, have reduced seizures or experience seizure freedom. ${ }^{89}$

The cortex responsible for generation of seizures may be resected or disconnected with epilepsy surgery to reduce or eradicate seizure, and surgery is the most effective treatment for certain groups of people with drug-resistant epilepsy. ${ }^{610}$ A limited number of individuals will nevertheless experience neurological, surgical and neuropsychological complications, ${ }^{11-13}$ but the risk of complication has reduced significantly to $<1 \%$ in recent years. ${ }^{14}$

Currently, surgical treatment is underutilised ${ }^{10} 1516$ despite the positive implications for reducing service use from overstretched services, improving patients' health and affirmative impacts on society. ${ }^{15} 1718$ One of the main contributors for underutilisation is said to be the delay in treatment. In the USA, for example, the period between onset of epilepsy and surgery can be up to 22 years. ${ }^{19} 20$ There are two different time periods of delays in treatment for resective surgery candidates. First, the initial onset and identification of refractory epilepsy to the referral for surgical assessment, and second, the period after patients have been referred for surgical assessment, to the time that they undergo surgical intervention, if they do so. Currently, in Australia, this can be anything from 6 months to 2 years. It is this second period of treatment delay that this study examines.

The critical gap of delay between initial identification of potential eligibility for surgery, surgical assessment and the eventual surgical intervention is said to affect the BoD for a substantial proportion of the epilepsy population and ongoing health service use and in-patient costs, pharmaceutical use, patient-professional communication, and work and interpersonal relationships. ${ }^{15}$

\section{Delays between assessment and treatment}

There are a number of challenges resulting from delays in epilepsy surgery. Delays may lead to social, psychological and medical comorbidities that would make surgical treatment difficult. Drug costs and costs of ongoing support are high, exacerbated by time from initial consultation to preparation for surgery and often multiple investigations and drug therapies along the way. Delays can leave patients indecisive, challenged by a multitude of decisions and lengthy negotiations over treatment and care, setting plans back for surgery or leading to no surgery at all. Consequently, the need for surgery can far outstrip the number of patients benefiting.

Clinical guidelines for both children and adults with epilepsy exist in the UK, ${ }^{21}$ Canada (2014) and the USA. ${ }^{22}$ While an international working group has supported a framework for future epilepsy-specific studies, ${ }^{23}$ the recommendations are broad by implication, and though adding weight to clinical practice in Australia, are not context specific, spanning a spectrum of areas, not only refractory epilepsy.
In the USA, clinical guidelines recommend, and benefits have been shown, for earlier identification and surgical intervention for refractory epilepsy. ${ }^{24}$ This has yet to be implemented in Australia. In view of the period of delay, resective surgery has been described as arguably the most 'underutilized of all accepted therapeutic interventions in the entire field of medicine ${ }^{, 19(\mathrm{p} .744)}$. At same time, resective surgery is recognised as: 'a powerful means of treating people with drug-resistant epilepsy', which: 'should not be viewed as a last resort'. ${ }^{25(\mathrm{p} .982)}$

\section{Understanding delay in assessment and treatment}

A range of factors may influence the treatment gap for patients with refractory epilepsy, such as patient concerns over social, psychological, physical and emotional long-term outcomes, consultation styles and lack of negotiated care. ${ }^{25}$ Prior international refractory epilepsy research has examined early intervention, ${ }^{8}$ drug use and effectiveness, ${ }^{26}$ intellectual disability, ${ }^{27}$ terminology for seizure grouping, ${ }^{28}$ ethnicity and socioeconomic factors ${ }^{29}$ psychosocial behaviour change and quality of life. ${ }^{30-32}$ In Australia, however, studies have yet to investigate the potential benefits of earlier intervention and the impact this can have on patients and healthcare professionals. We know of detailed research in Australia around the psychosocial impact of surgery, such as the difficulty of adjusting to seizure freedom, anxiety and depression, ${ }^{30}{ }^{31}$ Some anecdotal evidence suggests that the lack of resources in epilepsy centres and the lack of specialist epilepsy centres in the state of New South Wales (NSW) have also contributed to the delay. Other resource challenges may include long patient waiting lists, limited video electroencephalogram beds, and delays in receiving scans and neuropsychology results contribute. We now need better evidence to understand the factors that contribute to this delay and the effects delay has on patients, in order to improve patient health and well-being.

While clinical practice in other developed nations is driving forward initiatives to reduce the gap between initial identification, surgical assessment and surgical intervention, ${ }^{7} 1533$ changes are not being fully implemented in Australia. Examining the longitudinal treatment pathways and outcomes for patients, and whether or not surgery eventually takes place, will indicate how patients are assessed, how consultations occur, what affects people's views on surgery and the context in which delays happen. With the view to providing the best support and information possible to patients, and to improve the safety of healthcare services, this study will clarify the formation of patients' fears and assumptions around surgery and treatment. More evidence is needed to better inform the rationale for effective referral and close the gap in treatment for those who intend to take advantage of all the treatments available, including surgery. This in-depth examination of both hospitalisation data and patient interviews has not previously been conducted in NSW, Australia. 
Table 1 Study objectives outlined against data collection and patient outcomes examined

\begin{tabular}{|c|c|c|}
\hline Objectives & Data collection & Outcomes \\
\hline $\begin{array}{l}\text { Provide a qualitative account of treatment } \\
\text { and service provision and patient-reported } \\
\text { HRQoL (group 1) }\end{array}$ & $\begin{array}{l}\text { Observe consultations for communication } \\
\text { styles and treatment and service delivery } \\
\text { decisions } \\
\text { Semistructured interviews with patients and } \\
\text { healthcare professionals } \\
\text { Patient HRQoL questionnaires }\end{array}$ & $\begin{array}{l}\text { Patient and professional-reported } \\
\text { outcomes } \\
\text { Observational outcomes } \\
\text { Patient and healthcare } \\
\text { professional-reported outcomes } \\
\text { Patient-reported HRQoL }\end{array}$ \\
\hline $\begin{array}{l}\text { Comprehensive epidemiological study of } \\
\text { service use by patients with epilepsy across } \\
\text { New South Wales for a 5-year period } \\
\text { (group 3) }\end{array}$ & $\begin{array}{l}\text { Retrospective epidemiological data } \\
\text { assessment }\end{array}$ & $\begin{array}{l}\text { Service and treatment utilisation, } \\
\text { hospital separations, mortality, } \\
\text { medication use, clinical outcomes }\end{array}$ \\
\hline
\end{tabular}

HRQoL, health-related quality of life.

\section{STUDY OBJECTIVES}

The aim of this study is to examine the factors contributing to the delay of assessment and treatment for resective surgery candidates living with refractory epilepsy, and the effects delay has on patients. The objectives of the study are to:

1. Provide a qualitative account of treatment and service provision and patient-reported HRQoL.

2. Retrospectively review of medical records of patients who have undergone or have been assessed as candidates for resective surgery.

3. Comprehensive epidemiological study of service use by patients with epilepsy across NSW for a 5-year period.

The data collected and patient outcomes examined against secondary objectives are outlined in table 1.

\section{Study hypotheses}

Hypothesis 1: Patients' perceptions and experiences of clinical services and practices, surgical assessment procedures, and costs of drugs and other treatments, can affect the utilisation of surgery and reported HRQoL.

Hypothesis 2: Delays in surgical assessment and surgical intervention causes detrimental effect on the psychosocial health and well-being of patients.

Hypothesis 3: Health system issues such as resource use in hospitals and epilepsy centres is a factor of delay in offering surgical intervention.

\section{METHODS AND ANALYSIS}

\section{Study design}

This is a mixed methods study with (1) qualitative observations and interviews and HRQoL assessments for a group of 10 patients and six healthcare professionals (group 1). (2) A retrospective review of 50 patients' medical records from those attending two major epilepsy clinics, between 2014 and 2016 (group 2). (3) A retrospective, 5-year, epidemiological study of all health service use by patients with epilepsy across NSW (group 3).

This study uses mixed methods in order to most appropriately examine the BoD and treatment gaps for patients with refractory epilepsy, from initial patient identification to surgery and follow-up assessment, across the state of NSW, Australia. It will take place between 1January and 31 December 2017 with qualitative, HRQoL and patient record data collected from two of the major epilepsy clinics currently providing resective epilepsy surgery, in Sydney, and epidemiological data collected state wide, on all health service use by patients with epilepsy.

\section{Group 1 qualitative study}

The qualitative component of the study involves 16 participants and investigates treatment and clinical practices of patients with refractory epilepsy undergoing assessment for resective surgery. Ten separate consultations will be observed involving 10 patients managed by six different healthcare professionals, working across two epilepsy clinics in NSW. In addition, each patient-professional consultation that is observed will lead to interviews with the patient and healthcare professional in question, to gather views and opinions of interactions, treatment and care. Patient-reported HRQoL, at the point of care, will also be collected, via two standardised HRQoL assessment questionnaires: the 12-Item Short Form Survey and the EuroQol Five-dimensions-3-level questionnaire (SF12/ EQ5D3L).

\section{Group 2 medical records review}

This aspect of the study retrospectively assesses 50 patients' medical records from the same two epilepsy clinics in NSW. It will assess patients' demographic and clinical characteristics, and outcomes for those patients who have undergone, or were assessed as potential candidates for, resective surgery. 
Table 2 Study site and sampling for group 1 and group 2

\begin{tabular}{lcll}
\hline Study site & $\begin{array}{l}\text { Group 1 } \\
\text { Patients }\end{array}$ & $\begin{array}{l}\text { Group 1 } \\
\text { Healthcare } \\
\text { professional }\end{array}$ & $\begin{array}{l}\text { Group 2 } \\
\text { Patients }\end{array}$ \\
\hline $\begin{array}{l}\text { Westmead } \\
\text { Hospital }\end{array}$ & 5 & 3 & 25 \\
$\begin{array}{l}\text { Royal Prince } \\
\text { Alfred }\end{array}$ & 5 & 3 & 25 \\
Total & 10 & 6 & 50 \\
\hline
\end{tabular}

\section{Group 3 epidemiological study}

A retrospective, epidemiological study of individuals hospitalised with a diagnosis of epilepsy in NSW, in the most recent 5-year period (2012-2016), will take place, with data from approximately 11000 hospitalisations per year (55000 in total), assessed in order to examine health services use and treatment of individuals with epilepsy. This includes the health outcomes of individuals who underwent refractory surgery (group 3 ). The data drawn in group 3 will involve the linkage of NSW hospital separation and mortality data.

\section{Sample and setting}

\section{Group 1 and group 2 clinical setting}

The setting for group 1 and group 2 components of the study will be two neurology clinics in NSW. The neurology clinics have been chosen for their receipt of a substantial proportion of patient referrals for resective epilepsy surgery from across the state, and the clinics' ability to retain vital hospital data on patients who are being assessed and prepared for resective surgery, as well as those who have undergone surgical intervention. The study will draw 5 patients and three healthcare professionals from each clinic for group 1 data capture (total $\mathrm{n}=10$ patients and $\mathrm{n}=6$ healthcare professionals) and 25 patients' medical records from each clinic for group 2 data capture (total $n=50$ patients). See table 2 for details of study sites and sampling.

\section{Participants in groups 1 and 2}

Group 1 healthcare professionals

All consulting neurology clinicians, who work directly with patients with refractory epilepsy in a consultative role about assessment or surgery, in each epilepsy clinic, across the two public hospitals involved, will be invited to attend a study information meeting. During the meeting, these healthcare professionals will be given a full briefing of the aims, methods and intended outcomes of the study, in particular, stage 1 , and a study information pack. Their consent to participate in the study will be sought at the end of the meeting. Three healthcare professionals working most closely with patients with refractory epilepsy, whose main role is patient consultation through assessment for surgery or surgical intervention, will be consented to the study at each site.
Group 1 patients

Patients eligible for the study will be those treated by the same healthcare professionals involved from the two clinics. Those with refractory epilepsy will be identified by the clinical leads in each site. Recruitment will be according to timeframe sampling, where patients attending consultations will be invited to participate in consecutive order of consultation, from the commencement of the recruitment period, until the recruitment period ends, thus removing clinician bias and researcher coercion, until five patients have consented to participate from each site. Patients aged 18 or over, attending the clinics and being currently assessed for resective epilepsy surgery, will be included in the study. Patients who are under 18, who are unable to speak English and who are not willing to consent to the study will be excluded.

\section{Group 2 patients}

Fifty patients who are 18 years or older, assessed as suitable for resective epilepsy surgery during 2014, and consulting about surgery and other treatments for refractory epilepsy, across the two clinics involved, between 1 January 2014 and 31 December 2016, will be included in group 2. They will be randomly chosen from the complete 2014 cohort who were seen at each clinic, and once randomisation has identified the cohorts, access to their medical records will be obtained by a named Clinical Research Fellow. There will be one Clinical Research Fellow in each of the two clinics responsible for accessing patient medical records. A list of random numbers from which to choose the 25 patients' records to review, based on 300 new patients with refractory epilepsy at each clinic each year (the approximate total number of new patients attending), will be generated by the study team and provided to the Clinical Research Fellow, to aid random sampling.

The planned sequence of study visits and procedures for group 1 and group 2 study participants is outlined in figure 1.

\section{Group 3 data linkage and sampling}

Group 3 epidemiological study will involve the linkage of NSW Registry of Births Deaths and Marriages (RBDM) and the Cause of Death Unit Record File (COD URF) mortality data to the NSW Admitted Patient Data Collection (APDC) for the period 1 January 2012 to 31 December 2016. Case inclusion criteria for the group 3 study is all hospitalisations of individuals with epilepsy in the hospitalisation data between 1 January 2012 and 31 December 2016, with a hospital separation date during the same period. Hospitalisations will be identified using a diagnosis code of epilepsy in the APDC in the principal diagnosis or any of the 55 additional diagnosis records (ie, International Classification of Diseases (ICD)-10-AM: G40). Refractory epilepsy will be identified using the fifth character subdivision for category G40. We feel G40 is likely, if anything, to overdiagnose and thus can be assured, using this code, of not missing epilepsy. A 1-year 

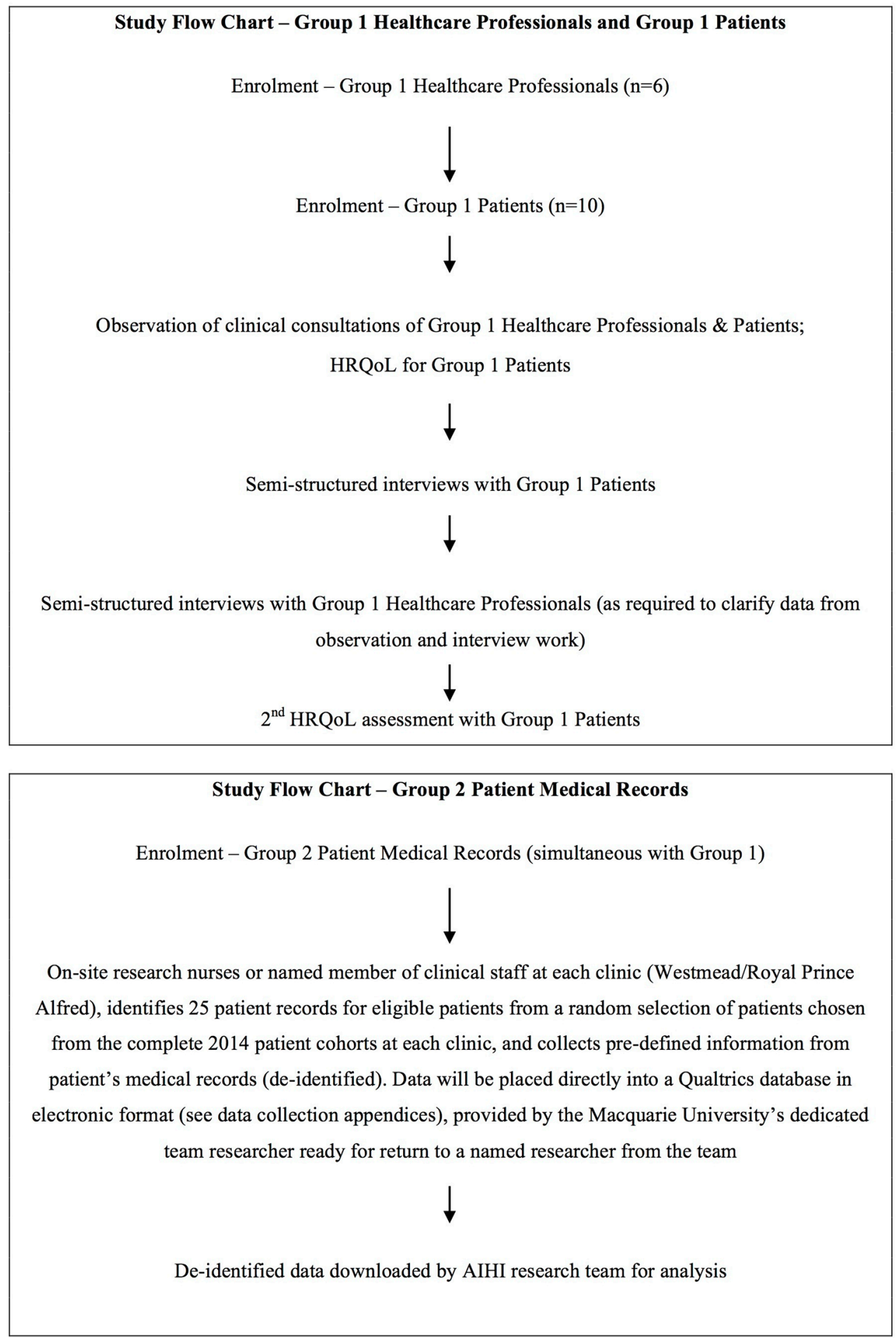

Figure 1 Study visits and procedures schedule. AlHI, Australian Institute for Health Innovation; HRQoL, health-related quality of life.

look-back period will also be used to inform the identification of any comorbidities, so the look-back period will extend the data to 1 January 2011. Individuals who undergo resective surgery will be identified using the classification: ICD-10-AM: 40 703-02 'Partial lobectomy of brain', which, in combination with epilepsy diagnosis, will verify a procedure was conducted for epilepsy reasons. Patient postcodes will enable consideration of patient spread across urban, rural and remote locations to take place.

\section{Data collection}

Data capture: group 1

Twenty-six qualitative data capture events are planned with patients across two clinic sites. This includes 10 observational events and 16 semistructured interviews. Qualitative 


\section{Box 1 Clinical observation topic guide}

Epilepsy consultation observations will concentrate on:

1. The context and timings around discussions about resective surgery treatment

2. Issues that are most concerning to patients and healthcare professionals

3. How treatment decisions are communicated and made

4. Discussions about timelines and delays in treatment

5. Patient-professional interaction, non-verbal communication

6. Professionals' and patients' views on patient pathways from presurgery to postsurgical care.

data collection is dependent on in-depth examinations of people's perceptions and behaviours and where qualitative studies combine observational and interview data, sample sizes are reduced to account for the extensive data that will be acquired in visual and textual formats. ${ }^{34}$ Based on previous research by team members, ${ }^{35}$ we require 26 observational and interview events to achieve data saturation in the prescribed sample set. Observations of 10 routine clinical consultations with patients with refractory epilepsy and their consulting neurology clinicians will take place. Observation is a technique that allows a third party, not directly involved in an event being observed, to gather an independent perspective. The observer will take note of, for example, the interactive process, and will also make deliberate observations of the multifaceted nature of the 'social worlds' within which the interactions takes place. ${ }^{36}$ This method will enable the examination of (1) the timing of discussions, (2) the language used by patients and healthcare professionals, (3) perceptions of treatment options and (4) intentions expressed. See box 1 for the clinical observation topic guide to be used in this study.

Semistructured telephone interviews will be undertaken with the same patient cohort according to a predesigned interview schedule. The healthcare professional cohort will be interviewed, to help further clarify observations and patient interviews. Interviews will illicit rich and detailed information to enhance the observer's field notes, ascertaining (1) how treatment decisions are made, (2) how surgery and surgical assessment is discussed, (3) discussions about timelines and delays in treatment and (4) healthcare professionals' views of patient pathways, from initial patient identification to postsurgical care. Interviews will be audiorecorded, and transcribed verbatim (de-identified to retain confidentiality), using external transcription services. See box 2 for the semistructured interview topic guide to be used in this study.

HRQoL assessment of patients' perceptions of HRQoL at the point of care will be collected via standardised quality-of-life assessment questionnaires (SF12/EQ-5D-3L). ${ }^{3738}$ Study participants will be asked to complete the questionnaires on the same day as their consultation, either prior to seeing the neurologist/epilepsy specialist or
Box 2 Semistructured interview topic guide

Patients in group 1 will be interviewed on the following topic:

1. Views on surgical assessment and treatment for refractory epilepsy including surgery

2. Timelines and delays in treatment

3. Sources of information

4. Role in decision making

5. Negotiations with healthcare professionals (and discussions with others, eg family)

6. Experience of surgery (if applicable)

7. Quality of life and psychosocial well-being

8. Plans for the future

Healthcare professionals in group 1 will be interviewed (if this will add clarity to patient interview data) on the following topic:

1. Views on surgical assessment and treatment for refractory epilepsy including surgery

2. Timelines and delays in treatment

3. Patients' concerns about surgical assessment and treatment

4. Negotiations with patients (and discussions with others, eg family/ healthcare professionals)

5. Quality of life and psychosocial well-being of patients

6. Views on postsurgical care

immediately after the consultation, to accommodate individuals' needs and waiting times. The HRQoL data will be used to contextualise, support and corroborate qualitative data collected in the interviews and observations, and will give insights into important physical and mental healthcare aspects of patient experience of epilepsy. ${ }^{39} 40$ The survey will be repeated at least 3 months postbaseline, depending on patient follow-up clinic appointment dates, for comparative purposes.

\section{Data capture: group 2}

Two Clinical Research Fellows will collect de-identified patient data directly from the randomly chosen patient records into an online, secure database, using the Qualtrics Survey Platform. All participants involved will be anonymised through a de-identification of medical records numbers from patient medical records by the two staff members responsible for data capture at each clinic. The survey questions comprise patient demographic information, diagnostic tests and presurgery assessment, surgical options and medication information (see Box 3: Medical Records Review Survey). Information will be collected for each visit during the study period (20142016). De-identified data, collected during the medical record review, will be downloaded and analysed by the study team.

\section{Data capture: group 3}

Data from the APDC include information on NSW inpatient separations (both public and private hospitals). The APDC contains information on patient demographics, source of referral, diagnoses, separation type, Australian 


\section{Box 3 Medical Records Review Survey}

\section{Medical Record Review Survey to be used by nurse researchers/healthcare staff member for reviewing medical records of} patients enrolled in group 2.

Q1 De-identified reference number

Q2 Age in years

Q3 Postcode

Q4 Marital status

1. Married/de facto relationship

2. Single

3. Divorced

4. Separated

5. Never married

6. Not known

Q5 Current driving status

1. Eligible to drive

2. Not eligible to drive

3. Not known

Q6 Employment status

1. Employed full time

2. Employed part time

3. Unemployed looking for work

4. Unemployed not looking for work

5. Retired

6. Student

7. Disabled

8. Not known

Q7 Main diagnosis

Q8 Secondary diagnoses

Q9 Date of referral to clinic ( $\mathrm{dd} / \mathrm{mm} / \mathrm{yyyy})$

Q10 Number of years since epilepsy diagnosed

Q11 Date of first visit during study period ( $\mathrm{dd} / \mathrm{mm} / \mathrm{yyyy})$

Q12 Surgery options at first clinic visit

1. Surgery not discussed or raised

2. Surgery discussed

3. Decision made to proceed to surgery

4. Decision made not to proceed to surgery

5. Side effects of surgery discussed

6. Referral to surgical team

7. Surgery completed

8. Postop visit

Q13 Tests ordered at first clinic visit

1. Blood tests to review drug levels

2. EEGs

3. Preop work-up

Q14 HRQoL tests on first clinic visit

1. General Anxiety Disorder (GAD) seven score

2. Neurological Disorders Depression Inventory for Epilepsy (NNDI-E) score

Q15 Please list epilepsy medications being taken by patient at first clinic visit (please list generic names where possible)

Q16 Medication management at first clinic visit

1. Number of medications changed

2. Number of medications where dosage was changed

Q17 Was there a second clinic visit during the study period

1. Yes

2. No

If No is selected, then skip to end of survey

Q18 What was the date of second clinic visit during study period ( $\mathrm{dd} / \mathrm{mm} / \mathrm{yyyy}$ )

Q19 Surgery options at second clinic visit

1. Surgery not discussed or raised

2. Surgery discussed

3. Decision made to proceed with surgery 


\section{Box 3 Continued}

4. Decision made not to proceed to surgery

5. Side effects of surgery discussed

6. Referral to surgical team

7. Surgery completed

8. Postop visit

Q20 Tests ordered at second clinic visit

1. Blood tests to review drug levels

2. EEGs

3. Preop work-up

Q21 HRQOL test on second clinic visit

1. GAD seven score

2. NNDIE score

Q22 Please list epilepsy medications being taken by patient at second clinic visit (please list generic names where possible)

Q23 Medication management at second clinic visit

1. Number of medications changed

2. Number of medication doses changed

Q24 Was there a third clinic visit during the study period

1. Yes

2. No

If No is selected, then skip to end of survey

Similar questions, about subsequent visits will be asked, if there were more than two visits.

EEG, electroencephalogram; HRQoL, health-related quality of life.

Refined Diagnostic Related Groups (AR-DRGs) and clinical procedures. Mortality data from the RBDM will provide information on fact of death, and cause of death will be identified using COD-URF. The AR-DRGs will be used to determine patient treatment and in-hospital healthcare costs. There is strong validity of hospital diagnosis coding in Australia. ${ }^{41}$

\section{Data analysis}

Group 1: qualitative data analysis

Field notes and interview transcripts will be analysed using thematic analysis techniques ${ }^{42}$ with two qualitative expert analysts (a primary and a secondary analyst) working together to ensure that the process is rigorous. Issues of significance will be noted through thematic analysis, with recurring concepts or ideas organised into common major and minor themes. ${ }^{42}$ The secondary analyst will examine a subsample of transcripts and field notes to build consensus around data veracity. All data will be considered corroboratively to develop a picture of the whole of the qualitative data capture activities, with data from interviews standing alongside researcher observations and HRQoL assessment results. The results of the qualitative data analysis will be used to map the gaps in treatment, and the perceptions and experiences of barriers to resective surgery from the perspectives of patients and epilepsy healthcare professionals.

\section{Group 1: HRQoL analysis}

Descriptive statistics will be used to describe the five dimensions and the visual analogue scale of the EQ-5D-3L: mobility, self-care, usual activities, pain or discomfort, and anxiety or depression. Spearman rank correlations will be used to assess correlation between the EQ-5D-3L overall health rating and subscales of the SF-12 for initial and follow-up questionnaires. Wilcoxon signed-rank test will be used to analyse the visual analogue scale and McNemer tests for the EQ-5D dimensions.

Results from the SF12 and EQ-5D-3L surveys will be converted into health utility index values using Australian specific value sets and the overall self-rated health status scores. ${ }^{3738}$

\section{Group 2: descriptive quantitative data analysis}

The results of the de-identified medical record reviews will be downloaded from a secure website by permissioned study staff. The most important outcome of this aspect of the study will be a detailed timeline for each patient identifying dates for initial referral, presurvey assessment, decisions relating to undergoing surgery and date of surgery (where performed). Descriptive analysis will examine associations of demographic and clinical indicators from the medical records, with surgery outcomes.

\section{Group 3: epidemiological data analysis}

The first epilepsy-related hospital admission during the 2012-2016 timeframe will be identified as the index epilepsy admission'. All hospital admissions and mortality records post the index epilepsy admission will be linked. Descriptive statistics will describe the number and characteristics of individuals with epilepsy and also with 'complex' epilepsy (including refractory epilepsy) who were hospitalised. Regression models (ie, linear, logistic, binomial/ Poisson) will be used to compare factors associated with epilepsy surgery and health outcomes (ie, hospital length of stay, readmission to hospital within 28 days and 30-day mortality posthospital admission). Descriptive statistics is the most appropriate method for reporting treatment 
cost data such as sum, mean, median, SD and total overall cost for key patient characteristics, such as epilepsy type, procedure, age group, sex, Intensive Care Unit admission and hospital length of stay.

\section{ETHICS AND DISSEMINATION}

Ethical approval for the study has been granted by North Sydney Local Health District Human Research Ethics Committee and the NSW Population \& Health Services Research Ethics Committee, along with Site Specific Assessments Approvals at each clinic.

\section{For group 1 healthcare professionals}

All healthcare professionals eligible for the study identified through the recruitment procedure described earlier (see Healthcare Professional Sampling) will be asked to sign a written consent form as a sign of willingness to participate.

\section{For group 1 patients}

Once healthcare professionals are recruited to the study, they will be asked to identify eligible patients to participate (see inclusion criteria) from their consulting lists, with the support of a dedicated Clinical Liaison Officer at each study site. Patients will be contacted via a letter accompanying their clinical appointment letter, to present the study to them and ask them to consider taking part. The request will be accompanied by a study information sheet and consent form that can be sent out by post or electronically, or handed to patients. Patients will be required to sign and return the consent form, or bring it to their planned consultation. There will be adequate time between receipt and consultation appointment to read and clarify queries from the patient information sheet. Once consented, the Clinical Liaison Officer will inform the study researcher of details of the planned visit, so as to plan for observations of consultations.

\section{Group 2 medical records review and group 3 epidemiological study}

A waiver of consent has been approved by the North Sydney Local Health District Human Research Ethics Committee and the NSW Population \& Health Services Research Ethics Committee for participants in groups 2 and 3, whose data will be de-identified prior to being provided to the study team for analysis, as per waiver of consent guidelines in the National Statement on Ethical Research, and the Research Exemption Statutory Guidelines. ${ }^{43}$

\section{Data storage and protection}

All electronic data will be stored in password-protected computers belonging to the university linked to the study team, and all hard copies will be stored in locked cabinets within secure offices. All data will be stored securely, and only the named researchers from the team will have access to the data.

\section{Participant feedback}

A plan is in place for study findings to be made available to participants in an executive summary document containing relevant information available about study processes, formatted for general consumption.

\section{Dissemination}

All data outputs will be disseminated as widely as possible, through academic and generalist publications and presentations. Dissemination may include methodological development, topic insights, gaps between patient identification and surgical treatment, and other therapies for severe epilepsy. Oral and poster presentations will be prepared for scientific conferences and other meeting fora.

\section{SIGNIFICANCE AND IMPACT OF THE STUDY}

This study is significant in being able to provide information on treatment decisions and other clinical practices for patients with refractory epilepsy undergoing, or being assessed for, resective surgery in Australia, and patients' and healthcare professionals' experiences and outcomes during the period 2014-2017. The study will provide robust evidence for the $\mathrm{BoD}$, and burden of treatment in patients with refractory epilepsy.

This is the first Australian study to examine implementation issues for resective surgery for patients with refractory epilepsy and to follow a cohort of patients through from their initial assessment, up to and including, surgical intervention. It will clarify information that is sorely needed, indicating which patients do and do not have surgery and the implications of this, and it will create a comprehensive picture of patients' health service use and medication use, over time. While this research will be undertaken using health data from NSW, it will identify differences between metropolitan areas and reveal socioeconomic differences between patients accessing healthcare services.

This study will inform a larger scale, pan-Australia study which will include socioeconomically, culturally, linguistically and geographically diverse patient cohorts to further examine the gap in treatment and the range of clinical practices for patients with refractory epilepsy. It will also elaborate cross-sectoral consultation in order to develop key clinical tools to enhance implementation of early identification and surgical intervention for refractory epilepsy. ${ }^{44}$

The study will be the first of its kind to comprehensively undertake a 5-year, retrospective epidemiological examination of health service use by patients with epilepsy, alongside a year-long qualitative, in-depth assessment of journeys by patients with epilepsy through the healthcare service, across key NSW clinics. The level of detail that will be disclosed will lend itself to data transferability to other jurisdictions. The study brings together a multidisciplinary team of epilepsy specialists, primary care practitioners, health services researchers, expert 
qualitative and quantitative methodologists, data-linkage researchers and health economists, to ensure greater understanding of health service provision in the wider Australian context and beyond.

Contributors FR led the overall conceptualisation of the study design, and led the first draft and revising of the manuscript. PS substantially contributed to the study design, led the ethics approval procedures, and contributed to the literature review and revising of the manuscript. RM conceptualised and led the design of the data linkage and epidemiological aspect of the study, and contributed to the first draft and revising of the manuscript. AN substantially contributed to the study design with epilepsy clinical expertise, and contributed to the first draft and revising of the manuscript. $A B$ substantially contributed to the study design with epilepsy clinical expertise, and contributed to the first draft and revising of the manuscript. GH substantially contributed to the study design with epilepsy clinical expertise, and contributed to the first draft and revising of the manuscript. SV provided primary care clinical expertise, and contributed to the first draft and revising of the manuscript. VM led the drafting of the patient records review survey work and the quality of life survey work, and contributed to the first draft and revising of the manuscript. All authors provided final approval of the version to be published and agreed to be accountable for the accuracy and integrity of the work.

Funding This work was supported by Macquarie University MQ Safety Net (MQSN) Grant Capture: Grant number: GT00503. Funding was also provided by the Royal North Shore Hospital Neurology to support epidemiological data capture.

Competing interests None declared.

Ethics approval Northern Sydney Local District Health HREC; NSW Population \& Health Services HREC.

Provenance and peer review Not commissioned; externally peer reviewed.

Open Access This is an Open Access article distributed in accordance with the Creative Commons Attribution Non Commercial (CC BY-NC 4.0) license, which permits others to distribute, remix, adapt, build upon this work non-commercially, and license their derivative works on different terms, provided the original work is properly cited and the use is non-commercial. See: http://creativecommons.org/ licenses/by-nc/4.0/

(c) Article author(s) (or their employer(s) unless otherwise stated in the text of the article) 2017. All rights reserved. No commercial use is permitted unless otherwise expressly granted.

\section{REFERENCES}

1. Laxer KD, Trinka E, Hirsch LJ, et al. The consequences of refractory epilepsy and its treatment. Epilepsy \& behavior 2014;37:59-70.

2. Edelvik A, Rydenhag B, Olsson I, et al. Long-term outcomes of epilepsy surgery in Sweden: a national prospective and longitudinal study. Neurology 2013;81:1244-51.

3. Devinsky O, Barr WB, Vickrey BG, et al. Changes in depression and anxiety after resective surgery for epilepsy. Neurology 2005;65:1744-9.

4. Trinka E, Bauer G, Oberaigner W, et al. Cause-specific mortality among patients with epilepsy: results from a 30-year cohort study. Epilepsia 2013;54:495-501.

5. Goldstein LH, McAlpine M, Deale A, et al. Cognitive behaviour therapy with adults with intractable epilepsy and psychiatric comorbidity: preliminary observations on changes in psychological state and seizure frequency. Behav Res Ther 2003;41:447-60.

6. Jobst BC, Cascino GD. Resective epilepsy surgery for drug-resistant focal epilepsy: a review. JAMA 2015;313:285-93.

7. Wiebe S, Blume WT, Girvin JP, et al. A randomized, controlled trial of surgery for temporal-lobe epilepsy. N Engl J Med 2001;345:311-8.

8. Kwan P, Schachter SC, Brodie MJ. Drug-resistant epilepsy. N Engl J Med 2011;365:919-26.

9. Spencer S, Huh L. Outcomes of epilepsy surgery in adults and children. The Lancet Neurology 2008;7:525-37.

10. Jehi L, Friedman D, Carlson C, et al. The evolution of epilepsy surgery between 1991 and 2011 in nine major epilepsy centers across the United States, Germany, and Australia. Epilepsia 2015;56:1526-33.

11. Georgiadis I, Kapsalaki EZ, Fountas KN. Temporal lobe resective surgery for medically intractable epilepsy: a review of complications and side effects. Epilepsy Res Treat 2013;2013:752195.

12. Rayner G, Wilson SJ. Psychiatric care in epilepsy surgery: who needs it? Epilepsy Curr 2012;12:46-50.
13. Rolston JD, Englot DJ, Knowlton RC, et al. Rate and complications of adult epilepsy surgery in North America: analysis of multiple databases. Epilepsy Res 2016;124:55-62.

14. Ryvlin P, Rheims S. Predicting epilepsy surgery outcome. Curr Opin Neurol 2016;29:182-8.

15. Engel J. Approaches to refractory epilepsy. Ann Indian Acad Neurol 2014;17(Suppl 1):S12):12.

16. Cloppenborg T, May TW, Blümcke I, et al. Trends in epilepsy surgery: stable surgical numbers despite increasing presurgical volumes. J Neurol Neurosurg Psychiatry 2016;87:1322-9.

17. Neurosurgical Associates. Epilepsy Surgery. http://www. neurosurgicalassociates.com.au/epilepsy.htm.

18. Rapport F, Storey M, Porter A, et al. Qualitative research within trials: developing a standard operating procedure for a clinical trials unit. Trials 2013;14:54.

19. Engel J. Overview of surgical treatment for epilepsy. The treatment of epilepsy. 3rd edn, 2009:741-56.

20. Berg A, Langfitt J, Shinnar S, et al. How long does it take for partial epilepsy to become intractable? Neurology 2003;60:186-90.

21. National Institute for Clinical Excellence. The epilepsies: the diagnosis and management of the epilepsies in adults and children in primary and secondary care. London: National Institute for Clinical Excellence, 2012.

22. Morris GL, Gloss D, Buchhalter J, et al. , Evidence-based guideline update: vagus nerve stimulation for the treatment of epilepsy: report of the Guideline Development Subcommittee of the American Academy of Neurology. Neurology 2013;81:1453-9.

23. Sauro KM, Wiebe S, Perucca E, et al. Developing clinical practice guidelines for epilepsy: a report from the ILAE Epilepsy guidelines Working Group. Epilepsia 2015;56:1859-69.

24. Kwan P, Brodie MJ. Clinical trials of antiepileptic medications in newly diagnosed patients with epilepsy. Neurology 2003;60(11 Suppl 4):S2-12.

25. Jetté N, Sander JW, Keezer MR. Surgical treatment for epilepsy: the potential gap between evidence and practice. Lancet Neurol 2016;15:982-94.

26. Glauser T, Ben-Menachem E, Bourgeois B, et al. ILAE treatment guidelines: evidence-based analysis of antiepileptic drug efficacy and effectiveness as initial monotherapy for epileptic seizures and syndromes. Epilepsia 2006;47:1094-120.

27. Kerr M, Gil-Nagel A, Glynn M, et al. Treatment of behavioral problems in intellectually disabled adult patients with epilepsy. Epilepsia 2013;54(Suppl 1):34-40.

28. Berg AT, Berkovic SF, Brodie MJ, et al. Revised terminology and concepts for organization of seizures and epilepsies: report of the ILAE commission on classification and terminology, 2005-2009. Epilepsia 2010;51:676-85.

29. Thompson AC, Ivey SL, Lahiff M, et al. Delays in time to surgery for minorities with temporal lobe epilepsy. Epilepsia 2014;55:1339-46.

30. Wilson SJ, Wrench JM, Mclntosh AM, et al. Profiles of psychosocial outcome after epilepsy surgery: the role of personality. Epilepsia 2010;51:1133-8.

31. Wilson SJ, Bladin PF, Saling MM. The burden of normality: a framework for rehabilitation after epilepsy surgery. Epilepsia 2007;48(Suppl 9):13-16.

32. Vingerhoets G. Cognitive effects of seizures. Seizure 2006;15:221-6.

33. Kerr M, Linehan $\mathrm{C}$, Thompson $\mathrm{R}$, et al. In response: evaluating the care of a multidisciplinary clinic by using the White Paper 'Listening for a change: Medical and social needs of people with intellectual disability who have epilepsy'. Epilepsia 2015;56:1473-4.

34. Creswell JW. Research Design: Qualitative, Quantitative and Mixed Methods Approaches. 2nd edn. California, USA: Sage Publications, 2003.

35. Rapport F, Clement C, Doel MA, et al. Qualitative research and its methods in epilepsy: contributing to an understanding of patients' lived experiences of the disease. Epilepsy Behav 2015;45:94-100.

36. Tracy SJ. Qualitative research methods: collecting evidence, crafting analysis. Communicating Impact. New Jersey, United States: John Wiley \& Sons, 2012.

37. The EuroQol Group. EuroQol-a new facility for the measurement of health-related quality of life. Health policy 1990;16:199-208.

38. Brazier JE, Roberts J. The estimation of a preference-based measure of health from the SF-12. Medical care 2004;42:851-9.

39. Stavem K, Bjørnæs H, Lossius MI. Properties of the 15D and EQ-5D utility measures in a community sample of people with epilepsy. Epilepsy Res 2001;44(2-3):179-89.

40. Birbeck GL, Kim S, Hays RD, et al. Quality of life measures in epilepsy how well can they detect change over time? Neurology 2000;54:1822-7. 
41. Henderson T, Shepheard J, Sundararajan V. Quality of diagnosis and procedure coding in ICD-10 administrative data. Med care 2006:1011-9.

42. Braun V, Clarke V. What can 'thematic analysis' offer health and wellbeing researchers? Int J Qual Stud Health Well-being 2014;9:26152.

43. National Health Medical Research Council. National statement on ethical conduct in human research: National Health and Medical Research Council, 2007.
44. Yamada J, Shorkey A, Barwick M, et al. The effectiveness of toolkits as knowledge translation strategies for integrating evidence into clinical care: a systematic review. BMJ open 2015 ;5:e006808

45. Rapport F, Clay-Williams R, Churruca K, et al. The struggle of translating science into action: foundational concepts of implementation science. J Eval Clin Pract 2017

(Epub ahead of print). 